\title{
Hacia un nuevo paradigma de salud laboral
}

\author{
Lucía Artazcoz $z^{a, b, c, d}$
}

DOI: $10.12961 /$ aprl.2019.22.01.1

En los últimos años los cambios económicos, sociales, técnicos y políticos han modificado profundamente la naturaleza del trabajo y el concepto de salud de la población trabajadora ${ }^{1}$. Sin embargo, en nuestro entorno la salud laboral sigue anclada en la evaluación y el control de los riesgos laborales y utilizando enfoques similares a los de hace décadas ${ }^{2,4}$.

\section{CAMBIOS EN EL MERCADO DE TRABAJO}

En los países desarrollados los riesgos laborales relacionados con las exigencias físicas han disminuido, mientras aumentan los riesgos psicosociales. Los cambios en la organización del trabajo se han acompañado de un aumento de los empleos atípicos, sobre todo el trabajo a tiempo parcial y el temporal. El primero ha pasado a ser, cada vez más, una forma de flexibilización interna de las empresas que, con frecuencia, dificulta la conciliación de vida laboral y familiar ${ }^{5}$, el segundo es una estrategia de flexibilización externa que igualmente permite a las compañías aumentar su competitividad. Ambos se asocian con peores condiciones de empleo y de trabajo y constituyen un riesgo potencial para la salud.

Se han producido cambios demográficos en la población trabajadora, más envejecida, feminizada y con una significativa proporción de personas inmigradas. La globalización ha supuesto una carrera contrarreloj para aumentar la competitividad de las empresas y de los países a costa de una desregularización generalizada del mercado de trabajo, la externalización de empresas, el abaratamiento de la mano de obra, el incremento de la incertidumbre laboral y de las desigualdades sociales y una pérdida de derechos entre la población trabajadora; en definitiva se ha generalizado la precariedad del empleo. La incorporación de las mujeres al mercado de trabajo cuestiona el modelo de salud laboral centrado exclusivamente en el trabajo remunerado y plantea la necesidad de un enfoque más amplio que incorpore la esfera doméstica y familiar y la distribución del trabajo reproductivo y del papel de persona sustentadora económica principal en el hogar ${ }^{6}$. En gran medida, como resultado de estos fenómenos, la distinción histórica entre exposiciones laborales y no laborales se ha vuelto artificial y menos útil para comprender los riesgos relacionados con el trabajo y desarrollar modelos de intervención de salud pública efectivos.
Abordar estos cambios requiere revisar en profundidad el modelo de salud laboral actual comenzando por considerar el trabajo, no solo como posible fuente de exposición a riesgos laborales - de seguridad, higiene, ergonómicos y psicosociales-, sino también como determinante social de la salud y uno de los principales mecanismos a través de los que operan las desigualdades sociales en la salud? ${ }^{7}$. Este nuevo paradigma también exige una visión centrada en el trabajo como fuente de salud, ya que el trabajo, o la falta de él, contribuye a posicionar a las personas trabajadoras y a sus familias en jerarquías sociales y es un determinante fundamental del poder, el dinero, el prestigio y el apoyo social y, por tanto, de la salud ${ }^{8}$.

En esta línea la OMS propone los siguientes objetivos para reducir las desigualdades en la salud relacionadas con el trabajo: 1) garantizar un alto nivel de empleo, de acuerdo con los principios de una economía sostenible, sin comprometer los estándares de trabajo decente y las políticas de protección social básica; 2) proteger los derechos laborales y aumentar los esfuerzos de prevención entre los grupos más vulnerables, especialmente las personas con contratos precarios, trabajadores y trabajadoras a tiempo parcial mal pagados, personas en paro e inmigrantes; 3) abordar el aumento del desempleo entre los jóvenes mediante la creación de oportunidades de empleo asegurando que ocupan un trabajo de alta calidad a través de políticas activas de educación, formación y de empleo y 4) mantener o desarrollar servicios de prevención de riesgos laborales financiados públicamente e independientes de los empleadores (aunque deben ser estos quienes los financien indirectamente) ${ }^{7}$. Este tipo de propuestas están muy lejos de las que la salud laboral clásica suele hacer, lo que evidencia el divorcio entre las prácticas de la salud laboral clásica y el abordaje del trabajo como determinante social de la salud.

A esta situación se suma que los sistemas de información actuales son inadecuados y no responden al objetivo de monitorizar la salud y sus determinantes en la población trabajadora, así como el impacto de las políticas en su estado de salud ${ }^{4}$. Finalmente, en comparación con otros ámbitos de la salud pública, la investigación en salud laboral es claramente insuficiente.

Autora para correspondencia:

Lucía Artazcoz

Correo electrónico: lartazco@aspb.cat b. CIBER de Epidemiología y Salud Pública (CIBERESP), Madrid, España.

c. Universitat Pompeu Fabra, Centro de Investigación en Salud Laboral,

Barcelona, España.

d. Institut de Recerca Biomèdica Sant Pau, Barcelona, España. 


\section{CLAVES PARA EL DESARROLLO DE UN NUEVO MODELO DE SALUD LABORAL}

A continuación se formulan propuestas agrupadas en tres líneas para una salud laboral capaz de dar respuestas a las nuevas necesidades de la población trabajadora.

\section{Redirección del foco hacia la salud, el bienestar y el trabajo}

Hasta ahora la salud laboral se ha centrado en la prevención de los riesgos laborales, es decir, ha puesto el foco en la enfermedad. Sin embargo, los marcos de los determinantes y las desigualdades sociales en la salud ponen el énfasis en la salud entendida en un sentido amplio que liga con el bienestar. La introducción del concepto de bienestar facilita la implicación de sectores distintos de trabajo y sanidad, y de la población general ${ }^{3}$ y, por tanto, el trabajo intersectorial. Este giro debería tener en cuenta los siguientes aspectos: - Inclusión del trabajo no remunerado en el modelo de salud, bienestar y trabajo, ya que no se puede entender la influencia del trabajo sobre la salud sin considerar la interacción entre los dos ámbitos ${ }^{6}$. - Incorporación del principio de "Salud en todas las políticas" en las decisiones organizativas de las empresas. Esto significa tener en cuenta el impacto de los cambios organizativos antes de llevarlos a cabo y su evaluación tras hacerlo.

- Incorporación del principio de "Salud en todas las políticas" en todas las decisiones políticas, más allá de las empresas, relacionadas, en mayor o menor medida, con el trabajo, desde la estimación del impacto en la salud, previa a la decisión sobre su implantación, a la evaluación del impacto en la salud.

- Desarrollo de políticas intersectoriales. El impacto del trabajo sobre la salud depende, no solo de las políticas de trabajo y de salud, sino de la gobernanza política y de las decisiones en otros sectores como las políticas económicas, de familia o de educación, lo que hace necesario impulsar políticas intersectoriales.

- Consideración del trabajo en un marco de determinantes y desigualdades sociales en la salud.

\section{Impulso de las políticas de conciliación de vida laboral y familiar}

Con la incorporación de las mujeres al mercado de trabajo se difumina la rígida división sexual del trabajo, pero todavía los hombres continúan sin compartir el trabajo doméstico y familiar y persisten claras desigualdades de género en el mercado de trabajo. En España, con un modelo de familia tradicional, a ellos se les asigna el papel de sustentadores económicos principales del hogar y los servicios públicos para el cuidado de personas independientes son absolutamente insuficientes. Estas son algunas de las actuaciones que se debería impulsar:

- Aumento de los servicios públicos a precios asequibles para el cuidado de niños y niñas de 0 a 3 años.

- Aumento de los servicios públicos a precios asequibles para el cuidado de personas dependientes: centros de día, ayuda domiciliaria y residencias para las personas con dependencia a cargo de trabajadores y trabajadoras.

- Implantación efectiva de los planes de igualdad de género en las empresas.
- Políticas de conciliación que dejen de partir de la premisa de que son las mujeres las responsables del cuidado de las personas dependientes, ya que esto las penaliza, tanto en el trabajo remunerado como en su salud y en su vida social'.

\section{Formación, información e investigación}

En la actualidad los sistemas de información en salud laboral son totalmente inadecuados y la formación en prevención de riesgos laborales es demasiado estrecha para los retos que suponen los cambios sociales y del mercado de trabajo. Además, la investigación en salud laboral es claramente insuficiente. Algunas modificaciones necesarias en este ámbito son las siguientes:

- Desarrollo de nuevos sistemas de información a partir de marcos conceptuales que señalen qué se debe medir, para buscar posteriormente las fuentes de datos, en contra de la estrategia frecuente de analizar de manera acrítica la información existente sin un cuerpo teórico que sustente el análisis. En este sentido podría ser interesante partir de marcos conceptuales generales como el de la Comisión de los determinantes sociales de la salud para el trabajo ${ }^{10}$.

- Impulso de colaboraciones académicas con distintas disciplinas para crear nuevos programas de docencia e investigación sobre el trabajo como determinante social de la salud (ciencias sociales, estudios laborales, negocios, economía, ciencias políticas, relaciones laborales y psicología laboral, por ejemplo). Entre las disciplinas de salud pública son importantes las alianzas con sectores relacionados con la epidemiología social y las desigualdades sociales en la salud ${ }^{4}$.

En definitiva se trata de avanzar hacia una convergencia de la salud laboral con la salud pública, ya que el trabajo es uno de los principales determinantes sociales de la salud. En la dicotomía en la que se suele mover la salud laboral entre la salud y los intereses económicos, este nuevo paradigma exige poner la salud y el bienestar de los trabajadores y trabajadoras en el centro de las decisiones.

\section{REFERENCIAS}

1. Wright MJ. The Changing Nature of Work. Am J Public Health. 2018;108:315-6. 2. Benach J, Muntaner C, Benavides FG, Amable M, Jodar P. A new occupational health agenda for a new work environment. Scand J Work Environ Heal. 2002;28:191-6.

3. Harrison J, Dawson L. Occupational Health: Meeting the Challenges of the Next 20 Years. Saf Health Work. 2016;7:143-9.

4. Peckham TK, Baker MG, Camp JE, Kaufman JD, Seixas NS. Creating a Future for Occupational Health. Ann Occup Hyg. 2017;61:3-15.

5. Chung H, Tijdens K. Working time flexibility components and working time regimes in Europe: using company-level data across 21 countries. Int J Hum Resour Manag. 2013;24:1418-34.

6. Artazcoz L, Cortès I, Escribà-Agüir V. Gender, work and health: a step forward in women's occupational health. In: Gideon J, editor. Handbook on Gender and Health. Cheltenham, UK: Edward Elgar Publishing; 2016.

7. Marmot M, Allen J, Bell R, Bloomer E, Goldblatt P. WHO European review of social determinants of health and the health divide. Lancet. 2012;380:1011-29.

8. Ahonen EQ, Fujishiro K, Cunningham T, Flynn M. Work as an inclusive part of population health inequities research and prevention. Am J Public Health. 2018;108:306-11.

9. Shankardass K, Muntaner C, Kokkinen L, Shahidi FV, Freiler A, Oneka G, et al. The implementation of Health in All Policies initiatives: A systems framework for government action. Heal Res Policy Syst. 2018;16:1-10.

10. Marmot M, Friel S, Bell R, Houweling TA, Taylor S. Closing the gap in a generation: health equity through action on the social determinants of health. Lancet. 2008;372:1661-9. 